\title{
HOME AUTOMATION USING INTERNET OF THINGS WITH SECURITY SYSTEM
}

\author{
M.Karthikeyan \\ Assistant Professor, \\ Department of EEE, \\ Vel Tech, Chennai, India,
}

\author{
S Prabakaran \\ UG Students, \\ Department of EEE, \\ Vel Tech, Chennai, India
}

\author{
P.Aravind \\ UG Students, \\ Department of EEE, \\ Vel Tech, Chennai, India
}

\author{
R.Vinoth Kumar \\ UG Students, \\ Department of EEE, \\ Vel Tech, Chennai, India
}

\begin{abstract}
In Current Situation, the Information and Communication Technology (ICT) are for the most part centered around the Internet of Things (IoT). In Day to Day life, IoT is one of significant piece of human life. Home computerization based IoT is flexible and prominent applications. The principle point of the undertaking is to build up a framework that will give remote control of home apparatuses and furthermore give protection from the disasters while home host isn't in home. This dissertation is primarily worried about the programmed control of light or certain another home machines utilizing web. This is intended to spare the electric power as well as human vitality. Its undertaking is done along the controller assistance and raspberry pi. The different apparatuses associated with the small scale controller and sensor is associated utilizing remote network. The proposed arrangement is sent and tried for different conditions. At long last, at this dissertation, our suggested arrangement working model is created into model as well as clarified as a working model.
\end{abstract}

Keywords-ICT, IoT, Home automation, Raspberry pi , Sensors, Smart home

\section{INTRODUCTION}

We are experiencing a daily reality such that is quickly advancing with respect to a Home at 21 st century will turn out to be increasingly more self-controlled as well as mechanized due to solace it gives, especially while used at private home. A home computerization framework represents which helps clients to control electric apparatuses of shifting kind. Several previous, entrenched home mechanization frameworks based upon wired correspondence. Its does not indicate a problem until the framework is organized in time as well as introduced when the physical improvement of the structure. Execution costs can be very high for previous structures (Atzori, Iera \& Morabito, 2010). In contrast, Wireless frameworks may incredible assistance for mechanization frameworks. Along the progression of remote inventions, such as Wi-Fi, cloud organizes at ongoing past, remote framework is used every day as well as everywhere. From little ventures to huge businesses mechanization this procedure is executed to diminish human work and to expand vitality proficiency. This process converting house into a smart house by using automation appliances like light, fan, temperature, smoke and we can add more as we want in future, this can be control by using IoT by any embedded system (Madakam, Ramaswamy \& Tripathi, 2015; Transpire online, 2019).

In this major things of home automation by remote control of home appliance and security when mishaps not in home. After the evolution of smartphones in modern world the growth of different communication technologies such as Wi$\mathrm{Fi}$, Bluetooth, ZigBee by this we can able to control or connect the home appliance when we need $t$ with help of need. We have many advantages by using home automation by conserving energy and saving the cost. In this with help sensor we have control smoke and temperature in this system we can add more in later (Bing, Fu, Zhuo \& Yanlei, 2011; Mythili et al., 2020).

In this paper the proficient home automation system is suggested. The important contribution of this dissertation is given below:

1) By controlling some home machines like fan, lights, smoke, and temperature by utilizing different sensors at various pieces of home.

2) Every sensors would associated with Node MCU ESP8266 or Raspberry pi that would process the readings gotten through sensors as well as regulate the transfers associated with apparatuses

3) The sensor is utilized to measure the temperature and smoke with help of IoT by keeping certain range in web page after it reach a certain range the alarm will be ring to control the temperature and smoke by controlling in web page.

4) The sensor is connected with Raspberry pi that kept control of home appliance and security system at time one device and it also can used to store in the cloud.

This dissertation is designed as given below. The previous home automation solutions are illustrated at Section II. Motivation is explained at Section III. Section IV explains the suggested work. Finally, section V concludes the paper. 


\section{RELATED WORK}

Home Automation is on the ascent at ongoing occasions. Beginning from farming, for urban communities having the high rises is slanted towards mechanization. At this area, we would examine different previous arrangements suggested through various research dissertations.

At (Madakam and Ramaswamy, 2014), TI Wi-Fi CC3200 Launch pad has is incorporated along PIR sensor for distinguish movement as well as forward a voice call to house proprietor. The proprietor would be able to choose whether to debilitate the security framework or not. He could likewise control household machines remotely. The execution additionally is next to no with respect to computerization.

At (Wang, Zhang, Zhang, Zhang and Li, 2013) the creator utilizes $433 \mathrm{MHz}$ radio recurrence control module to regulate the household apparatuses legitimately. Devices such as cell phones, tablets may legitimately associate with focal controller utilizing $\mathrm{Wi}-\mathrm{Fi}$ interface. The main issue of radio sign is it may effectively blocked also inclined for twists because of obstruction.

At (Kazi and Tiwari, 2015) creator utilizes the Blue tooth 4.0 convention for set up correspondence among "keen household machines" as well as client. To control these machines remotely the client may utilize mobile phones or tablets. The main impediment to utilizing Blue tooth innovation is to control gadgets from a short period of time. At (Piyare and Tazil, 2011), creator's suggested Blue tooth in terms of home computerization frameworks. At this arrangement, household machines were regulated dependent by Android cell phones without the controllability of Internet. Every household apparatuses were physically associated with the controller of Blue tooth also it constrained through utilizing Smartphone. Nonetheless, at this arrangement, household apparatuses do not remotely associated as well as it does not ready to work remotely.

Based on IoT, home mechanization utilizing android telephones (Mandula, Parupalli, Murty, Magesh and Lunagariya, 2015) is suggested. At this, creators utilized double sorts of home robotization, that is Blue tooth as well as Ethernet-depend. Additionally, modified Android-depend versatile utilization is utilized for regulating household utilizations such as Fan, TV, AC, and so on. In any case, information sources in the model isn't clear and it drives stop circumstance when numerous Android telephones attempt to get to same online interface.

In (Gupta and Chhabra, 2016) a shrewd information obtaining framework and vitality the board framework has been made which shows the important information in website page along the assistance of SMS, GPRS, email alarms. ARM microcontroller is utilized to control the gadgets as well as to remote correspondence. Framework is planned dependent upon IEEE1451 convention that spotlights in characterizing electronic information sheet of transducer to every one of the transducers utilized.
Ease, adaptable as well as pervasive brilliant home framework was exhibited (Kumar, 2014). At this point, Arduino Ethernet was utilized to impart over the Internet along redid utilization introduced on the Android portable. The Arduino gadget is coordinated along different sensors such as dampness, temperature, smoke/gas. Every one of such sensors was constrained through versatile utilization utilized through client. Suggested model is tried as well as consists of examined the productivity. Nonetheless, when the specific home utilization arrives at the given limit, suggested framework created alarms at structure email messages.

At (Kodali, Jain, Bose and Boppana, 2016) Ethernetdepend savvy home mechanization as well as vitality the executives framework is suggested dependent upon the Intel Galileo advancement board. Different sensors are utilized to regulate the home machines just as to keep up safety of home.

At (Madakam and Ramaswamy, 2014) TI Wi-Fi CC3200 Launchpad is coordinated along PIR sensor to distinguish movement as well as send a voice call to house proprietor. The proprietor would be able to choose whether to debilitate the security framework or not. The proprietor could likewise control the household machines remotely. This usage likewise is next to no with respect to robotization.

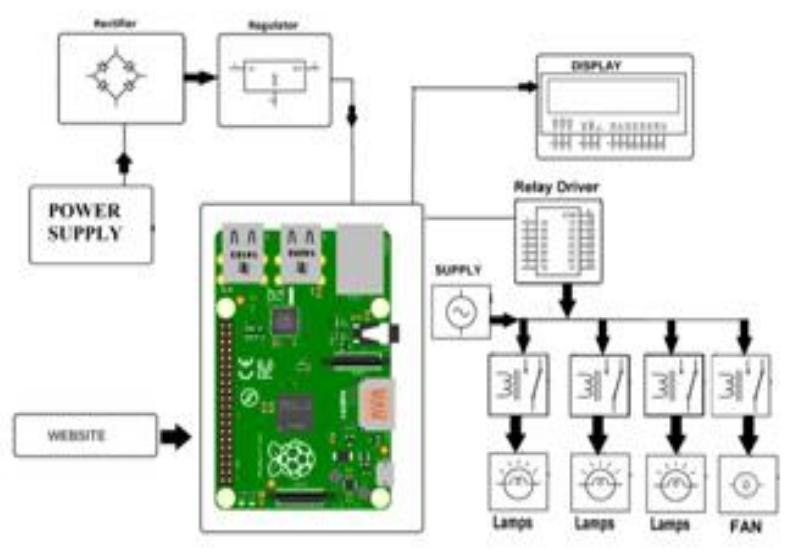

Fig.1. Suggested System Configuration

\section{Motivation}

Home automation is rise over for previous couple of years. Along regularly developing innovation, there are more intelligent as well as further developed arrangements at the space of home robotization. To improve the way of life, apparatuses should be entirely computerized with no client intercession in any structure at all. This empowers the end client bother free collaboration with the apparatuses as the machines learn and respond according to the client's necessities would physically squeezing a catch without him. Wired sensor framework is increasingly hard to deal with and furthermore need more measure of wired sensors in various areas. Accordingly, significance of remote sensor hub is in ascent as well as is the basic aspect to proficient usage of 


\section{International Journal of Engineering Applied Sciences and Technology, 2020 \\ Vol. 5, Issue 7, ISSN No. 2455-2143, Pages 152-156 \\ Published Online November 2020 in IJEAST (http://www.ijeast.com)}

home computerization. Vitality sparing is the critical focal points of mechanizing home machines. Accordingly, client should be keep mindful of vitality utilization of robotized apparatuses. At creating nation such as India, here individuals has occupied life plans, giving proficient vitality sparing as well as comfort in less costs also higher effectiveness is most extreme significance.

\section{PROPOSED SYSTEM}

The configuration of suggested system as well as prototype represents at Figure 1 as well as $\mathrm{Figure} 2$. The given sections illustrate about the working modules involved at the suggested solution.

\section{A. System Design}

Our suggested prototype primarily has three layers of implementation, namely:

\section{- Sensor Node Layer}

- Sensor Data as well as Database Interface Layer

- Server as well as Notification Layer

1. Sensor Node Layer: Sensors, to be specific, smoke sensor, and temperature sensor are associated with the Raspberry pi BCM2837. CT sensor, HX711 ADC module as well as heap cell is associated with raspberry combined along Ethernet shield. Sensors gain the information about the home condition factors as well as forward the information to BCM2837.

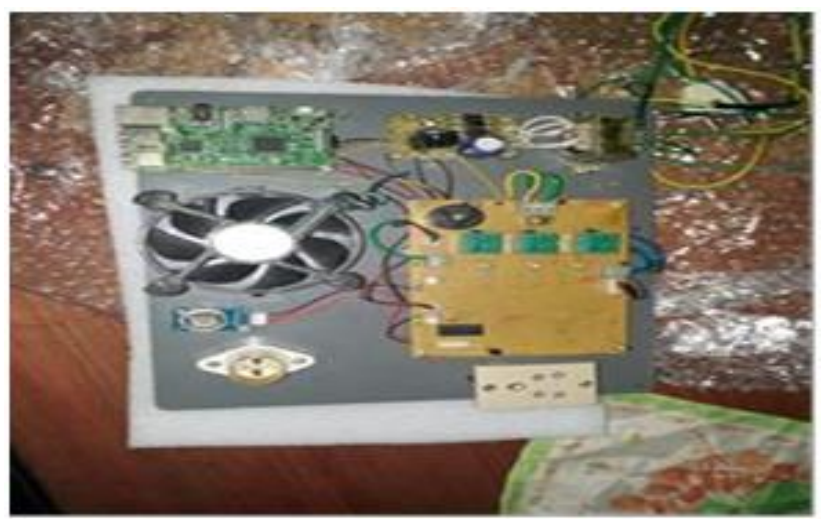

Fig.2. Proposed Prototype design

The BCM2837 at that point triggers the transfers associated with the lights, blinds, fan, climate control system temperature when vital conditions are met. A control gadget is microcontroller which is installed along peripherals, memory as well as processor. The remote controlling and computerization of the machines are made conceivable by microcontroller that is customized to process the reaction created by the different sensors and to trigger the apparatuses as per the robotization engineering. The two microcontrollers utilized in the undertaking are:

a) Raspberry Pi called charge card measured PC fueled through Broadcom BCM2835 framework on-a-chip (SoC). SoC integrates 32-piece ARM1176JZFS processor, timed in $700 \mathrm{MHz}$, Video center IV GPU. Likewise a POP bundle over the SoC consists of $256 \mathrm{MB}$ of RAM. Raspberry $\mathrm{Pi}$ is fuel through $5 \mathrm{~V}$ small scale USB AC charger or feasibly $4 \mathrm{AA}$ batteries (with the touch of hacking).

1) BCM2837: The BCM2837 is the less Wi-Fi chip effort along complete TCP/IP stack as well as the capacity of MCU (microcontroller unit) supplied by Shanghai-based Chinese maker, Expressive Systems. The ease, minimized size and the nearness of an inbuilt Wi-Fi module and Bluetooth are the purposes behind choosing the MCU.

2. Sensor Data as well as Database Interface Layer: The sensor of temperature as well as smoke coupled with the Raspberry with help relay. The obtained data is executed through Raspberry and as a with help IOT the smoke and temperature are kept in certain range. BY using web page we monitor the temperature and smoke level in constant range the structure of web page represents at fig 3

3. Server as well as Notification Layer: Mentioned above serve get the information with help of web page as shown sample web page of the system. In the proposed system we control the light and fan temperature, smoke by using IoT with help or web page .In this web page are created by us to control at from any place with help IOT.

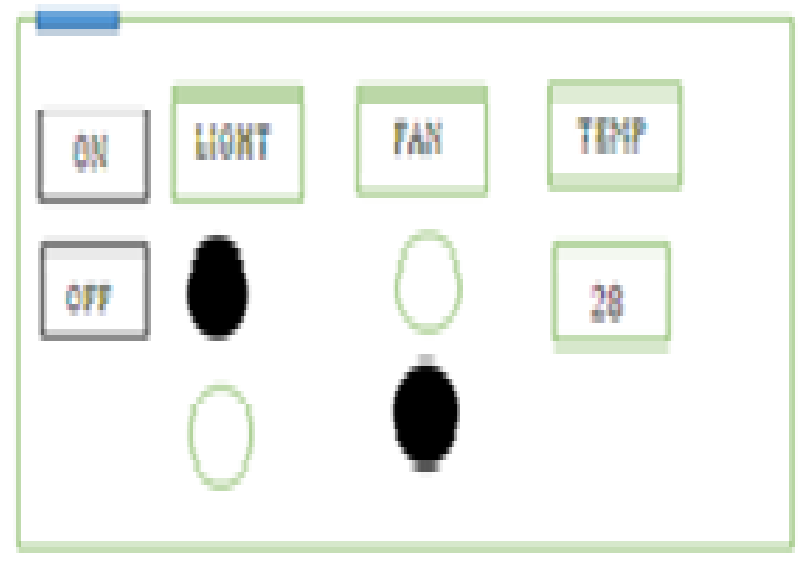

Fig.3. Sample web page structure

\section{B. Objectives}

The sensor hub detects the ongoing changes in every one of the factors at room utilizing different sensors. The microcontroller acclimatizes sensors in each room. It will control your home mishaps when you not in home if you want your ac on to kept at particular temperature it automatically keep the room temperature in certain level .

\section{Implementation Details}




\section{International Journal of Engineering Applied Sciences and Technology, 2020 \\ Vol. 5, Issue 7, ISSN No. 2455-2143, Pages 152-156 \\ Published Online November 2020 in IJEAST (http://www.ijeast.com)}

1) Data Flow Diagram (DFD): Working model is appeared as DFD as given in Figure 5 and clarified beneath:

- The sensors in the tangible hubs recognize the factors estimations in nature and send it to the converter of Analog to Digital.

- ADC transfers the changed over information to the microcontroller which procedures the acquired worth set.

- The microcontroller controls the transfers which are associated with fan and light contingent upon the sensor worth got.

- Then, the microcontroller flashes the data on the sequential screen which thus is sent to the database.

- A nearby server is made utilizing XAMPP to transmit the data send to website page to see it.

b) 2) Hardware: Raspberry $P i$ called charge card measured PC fueled through Broadcom BCM2835 framework on-a-chip (SoC). SoC integrates 32-piece ARM1176JZFS processor, timed in $700 \mathrm{MHz}$, Video center IV GPU. Likewise a POP bundle over the SoC consists of $256 \mathrm{MB}$ of RAM. Raspberry Pi is fuel through $5 \mathrm{~V}$ small scale USB AC charger or feasibly 4 AA batteries (with the touch of hacking).

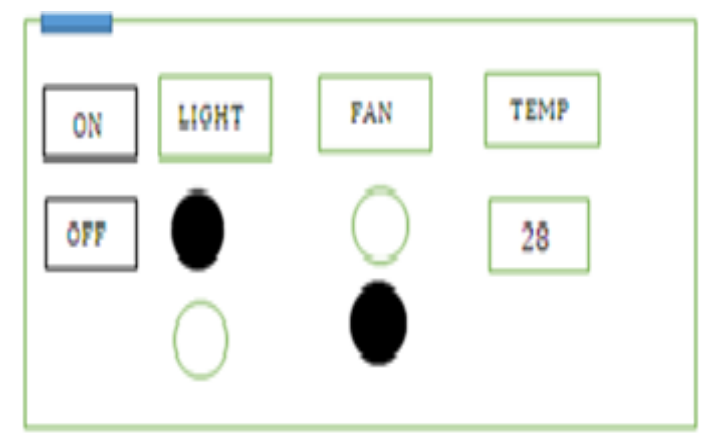

Fig.4. Web page

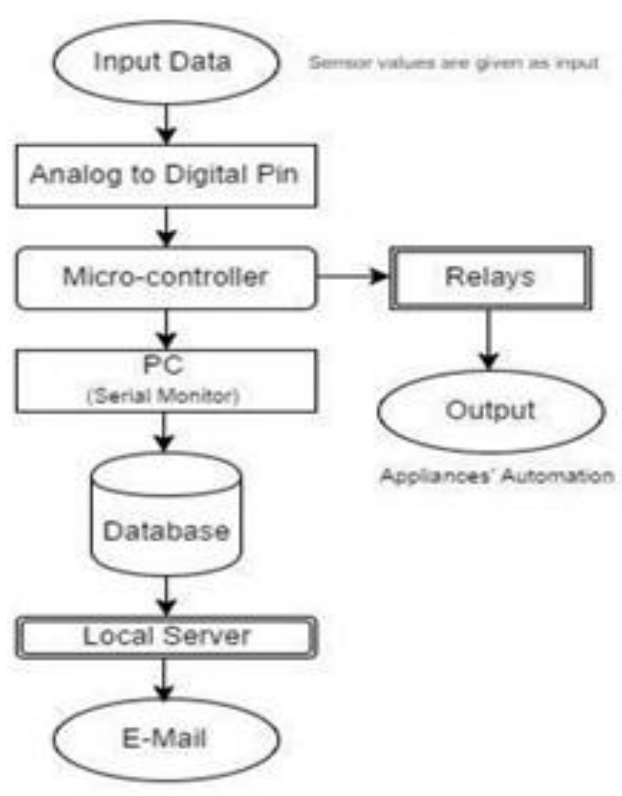

Fig. 5. Suggested system data flow diagram

3. Database and Server Programming: To make a nearby server of host PC is utilized XAMPP. In PHP as well as MySQL the database related programming is done. The sensor information is perused into the table made in MySQL is appeared in Sample database structure. To send the program customer data to the neighborhood server, we utilize the GET strategy. In this, the data is encoded utilizing a plan called URL encoding. The Raspberry program adds this encoded data to the page demand in this way sending this data to the PHP program where these qualities are put away in neighborhood factors. Utilizing SQL inquiries, these qualities are added into the database which is being controlled by PHPMyAdmin. The PHP program at that point advises the client about the units expended, and expected.

Algorithm 1 Node MCU ESP8266

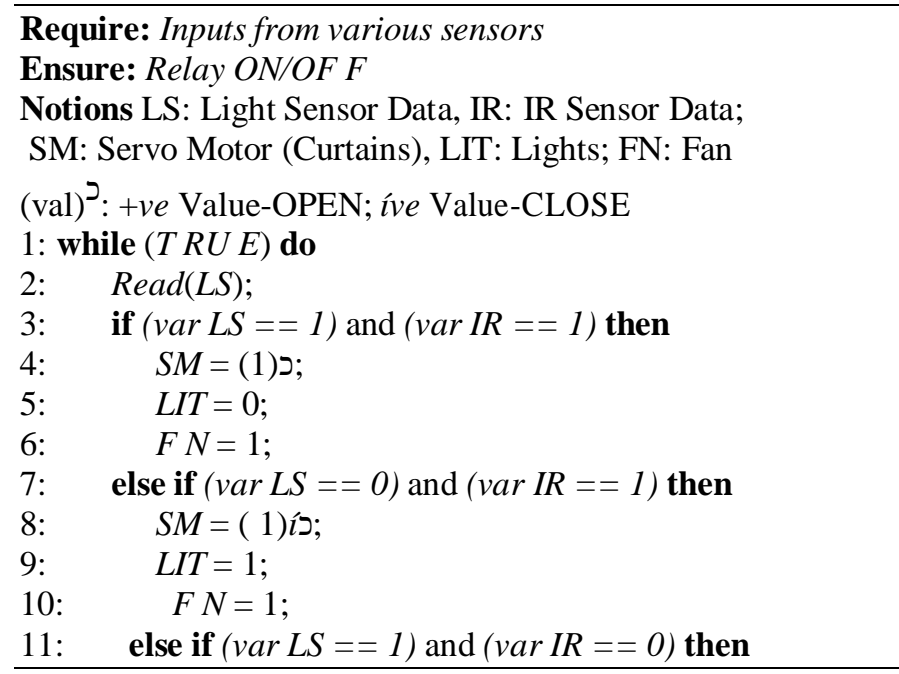




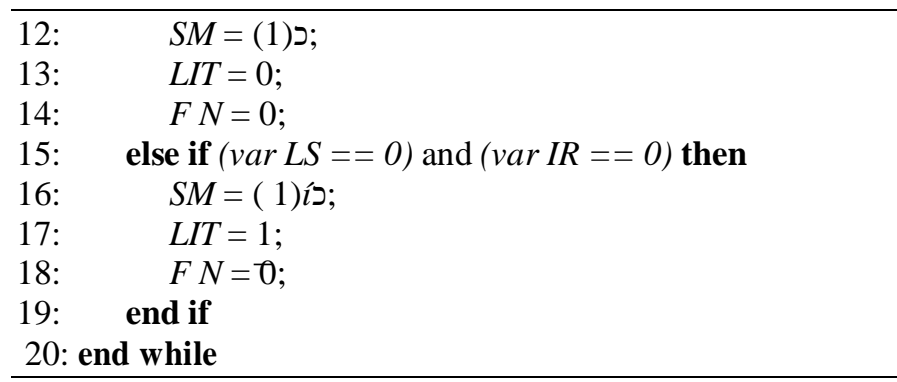

\section{CONCLUSION}

At this dissertation, through thinking about the wireless sensor hub the home computerization is enhanced. The keen house coordinates different electric machines at house also computerizes there with no or least client mediation. The brilliant house monitors diverse condition factors present and aides the apparatuses to work as indicated by the requirements of the client. Not just robotizing the home machines of day by day utilization yet additionally informing the client regarding cost of electric bill at normal interim as well as if gas degree arrives at less than the limit naturally reserving the gas chamber. Thinking about the highlights above, we built up as well as tried the model. Through utilizing the IoT innovations we accomplished the Smart Home advancement. From the test, it was discovered that we can figure out how to make minimal effort, adaptable and vitality proficient brilliant home for improving things and greener future.

\section{REFERENCES}

1 Atzori, L., Iera, A., and Morabito, G. (2010). The Internet of Things: A survey. Computer Networks, 54(15), 2787-2805.

2 Bing, K., Fu, L., Zhuo, Y., and Yanlei, L. (2011). Design of an Internet of Things-based smart home system. 2011 2Nd International Conference On Intelligent Control And Information Processing. vol. 2, 2011, 921-924

3 Gupta, P., and Chhabra, J. (2016). IoT based Smart Home design using power and security management. 2016 International Conference On Innovation And Challenges In Cyber Security (ICICCS-INBUSH), 6-10.

4 Kamal, M., Parvin, S., Saleem, K., Al-Hamadi, H., and Gawanmeh, A. (2017). Efficient low cost supervisory system for Internet of Things enabled smart home. 2017 IEEE International Conference On Communications Workshops (ICC Workshops). 864869

5 Kazi, R., and Tiwari, G. (2015). IoT based Interactive Industrial Home wireless system, Energy management system and embedded data acquisition system to display on web page using GPRS, SMS and E-mail alert. 2015 International Conference On Energy Systems And Applications, 290-295.

6 Kodali, R., Jain, V., Bose, S., and Boppana, L. (2016). IoT based smart security and home automation system. 2016 International Conference On Computing, Communication And Automation (ICCCA), 1286-1289.

7 Kumar, S. (2014). Ubiquitous Smart Home System Using Android Application. International Journal of Computer Networks and Communications, 6(1), 3343.

8 Madakam, S., and Ramaswamy, R. (2014). Smart Homes (Conceptual Views). 2014 2Nd International Symposium On Computational And Business Intelligence, 63-66.

9 Madakam, S., Ramaswamy, R., and Tripathi, S. (2015). Internet of Things (IoT): A Literature Review. Journal Of Computer And Communications, 03(05), 164-173.

10 Mandula, K., Parupalli, R., Murty, C., Magesh, E., and Lunagariya, R. (2015). Mobile based home automation using Internet of Things(IoT). 2015 International Conference On Control, Instrumentation, Communication And Computational Technologies (ICCICCT), 340343.

11 Piyare, R., and Tazil, M. (2011). Bluetooth based home automation system using cell phone. 2011 IEEE 15Th International Symposium On Consumer Electronics (ISCE), 192-195.

12 Wang, M., Zhang, G., Zhang, C., Zhang, J., and Li, C. (2013). An IoT-based appliance control system for smart homes. 2013 Fourth International Conference On Intelligent Control And Information Processing (ICICIP), 744-747.

13 A New Seagull Optimization Algorithm to Solve Numerical Optimization Problems, Transpire online (2019). Available at: https://transpireonline.blog/2019/08/30/a-newseagull-optimization-algorithm-to-solve-numericaloptimization-problems/. [Accessed on: 10 Jan 2020]

14 Mythili, S., Thiyagarajah, K., Rajesh, P., and Shajin F. H. (2020). Ideal position and size selection of unified power flow controllers (UPFCs) to upgrade the dynamic stability of systems: An antlion optimiser and invasive weed optimisation Algorithm. $\begin{array}{lll}\text { HKIE } & \text { Transactions. } & 27 .\end{array}$ 10.33430/V27N1THIE-2018-0024. 